\title{
APUNTES DE ECONOMÍA ORTODOXA A LA REGULACIÓN CONTABLE, ELEMENTOS PARA LA COMPRENSIÓN Y CRITICA DE LAS NORMAS CONTABLES*
}

\author{
JORGE ALEXANDER RODRÍGUEZ OTÁLORA** \\ UNIVERSIDAD LIBRE DE COLOMBIA-SECCIONAL CALI
}

Recibido/ Received/ Recebido: 17/05/2012 - Aceptado/ Accepted / Aprovado: 18/10/2012

\begin{abstract}
Resumen
La intencionalidad de este artículo es, desde el punto de vista de la economía ortodoxa, presentar los fundamentos de la regulación contable, estableciendo recorridos conceptuales que nos permitan comprender los supuestos que la fundamentan. Las categorías de mercado, agente, institución, norma, regla, entre otras, enmarcadas en las posturas neoclásica y neoinstitucional son el telón de fondo sobre el cual se analizará la contabilidad. Se revisan diferentes teorías que sustentan la regulación económica para comprender el proceso regulativo en sí. El escrito es de carácter introductorio, intentando brindar argumentos teóricos ortodoxos a la discusión acerca del proceso de convergencia contable.
\end{abstract}

Palabras clave: Regulación, Contabilidad, Economía neoclásica, economía neoinstitucional, Agentes.

\section{ORTHODOX ECONOMY NOTES TO ACCOUNTING REGULATION, ELEMENTS TO COMPREHEND AND CRITICIZE ACCOUNTING RULES}

\begin{abstract}
The purpose of this article is to present the fundaments of accounting regulation from an orthodox economic point of view; establishing conceptual paths that allow comprehending the fundamental assumptions. Market categories, agent, institution, standard, rule, among others, framed in neoclassic and neo-institutional schools are the background in which the accountability will be analyzed. Various theories are reviewed which support the economic regulation to comprehend the regulation process and its objectives. The article is introductory, and provides orthodox theoretical arguments to the discussion on accounting convergence process.
\end{abstract}

Keywords: Regulation, Accounting, Neoclassic economy, Neoinstitutional economy, Agents.

Artículo de reflexión derivado del proyecto de investigación "Una propuesta para el abordaje de la contabilidad financiera en los procesos de enseñanza"

** Contador público con maestría en Ciencias Económicas. Docente investigador Universidad Libre de Colombia. Seccional Cali. Correo electrónico: alexrodriguezo@hotmail.com 


\title{
ANOTAÇÕES DE ECONOMIA ORTODOXA À REGULAÇÃO CONTÁBIL, ELEMENTOS PARA O ENTENDIMENTO E CRÍTICA DAS NORMAS CONTÁBEIS
}

\begin{abstract}
Resumo
A intenção deste artigo é, a partir do ponto de vista da economia ortodoxa, apresentar os fundamentos da regulamentação contábil, estabelecendo trajetos conceituais que nos permitam compreender os pressupostos que a fundamentam. As categorias mercado, agente, instituição, norma, regra, entre outras, no contexto das posturas neoclássicas e neoinstitucional, são o pano de fundo sobre o qual se analisará a contabilidade. Revisam-se diferentes teorias que sustentam a regulamentação econômica para compreender o processo regulamentar em si e seus fins. O escrito é de caráter introdutório, tentando brindar argumentos teóricos ortodoxos à discussão a respeito do processo de convergência contábil.
\end{abstract}

Palavras chave: Regulamentação, Contabilidade, Economia neoclássica, Economia neoinstitucional, Agentes.

Rodríguez, A. (2012) Reflexiones sobre la regulación en contabilidad, una visión desde la economía ortodoxa. En: Revista de la Facultad de Ciencias Económicas de la Universidad Militar Nueva Granada. rev.fac.cienc.econ, XX (2).

JEL: B41, B52, M41.

\section{Introducción}

Es importante vincular el pensamiento económico a la regulación contable, observando los aportes e injerencias, más allá de las exigencias de registro e información de los entes económicos (organizaciones) que se hacen a través de la normatividad. Los supuestos económicos base de la corriente dominante unido a los desarrollos sociológicos han ampliado $e$ intensificado la presencia de un pensamiento único frente a las relaciones sociales, económicas y contables. Así es necesario develar las implicaciones de su uso en la regulación contable para, desde allí, dar atisbos de comprensión de los fenómenos en que la contabilidad se ve envuelta.

Este escrito pretende ser un preámbulo para la comprensión de una problemática que ha estado presente a lo largo del tiempo tanto en la disciplina como en la práctica de la contaduría, la incidencia de la contabilidad en los fenómenos organizacionales y sociales. Para ello se presenta una serie de argumentos de origen económico ortodoxo que permiten ubicar la regulación contable en un espectro amplio, vinculándola con la racionalidad de los agentes, el mercado y en general con el conjunto de relaciones sociales que hacen parte de las dinámicas económicas, sociales y ambientales.

Revisar las diferentes teorías que sustentan la regulación económica es importante para comprender el proceso regulativo en sí y los fines que busca. El carácter introductorio del escrito pretende brindar argumentos teóricos ortodoxos a la discusión acerca del proceso de convergencia contable, para que la discusión de argumentos heterodoxos encuentre una comunidad contable más informada y así contribuir al esclarecimiento de diversas posturas que confluyen cuando se habla de regulación en contabilidad.

Se aborda la regulación económica como forma en la cual se organiza el sistema económico desde la óptica neoclásica y neoinstitucionalista. Posteriormente se observa cómo son las decisiones políticas entre la conveniencia de seguir una línea teórica, (ya sea el interés público o interés privado), las que definen la construcción de los mecanismos de la regulación. Un factor necesario en la discusión es la racionalidad de los agentes ¿cómo es entendida para los regulado- 
res? para saber las pretensiones que tienen frente al nivel de incidencia de la regulación en los agentes. Con lo anterior como telón de fondo se analizará la información que brinda la contabilidad como bien público y sus interacciones teóricas internas y externas, llevando a una visión preponderante de la regulación contable que a luz de las nuevas problemáticas sociales y ambientales se hace insuficiente.

\section{Regulación económica, ¿el problema del funcionamiento del mercado?}

La principal fundamentación teórica de la regulación económica se basa en la teoría neoclásica, que desde un planteamiento liberal clásico aborda la regulación en su mínima expresión, teniendo en cuenta que son los mercados y el sistema de precios, los que permiten la relación entre los diversos agentes. Además, para comprender mejor los elementos de la regulación económica, se deben tener en cuenta las hipótesis que según Cataño (2001) describen este sistema económico:

- Los agentes se definen por sus dotaciones de bienes físicos y derechos sobre las ganancias de las firmas, sus preferencias y sus dotaciones iníciales

- Existe un conjunto de producción disponible para las firmas dadas.

- Todos los agentes tratan los precios paramétricamente (precios relativos).

- Todos los agentes son racionales.

- Mercado y sistema de precios son los mecanismos de relación entre los individuos.

En esta tradición se considera que los agentes (firmas) privados son los encargados de la asignación de los recursos, producción y distribución, por lo que la intervención pública sólo debe hacerse en casos muy particulares, laissez faire. El equilibrio general se presenta como la solución más general al problema de la mano invisible: múltiples agentes, mercados en competencia perfecta capaz de determinar un criterio individualista de eficiencia (Cataño, 2001). Sin embargo y sin salirse de esta tradición, varios economistas han demostrado que el mercado no funciona bien por sí mismo, necesita ayuda para garantizar su funcionamiento (Greenwald \& Stiglitz, 1986; Stiglitz, 2006; Krugman, 2009).

En ese escenario, es donde el Estado o las instituciones independientes, entran a ejercer control a las actividades económicas, estableciendo una serie de incentivos coactivos o coercitivos a los agentes para que actúen de determinada manera, en pro del funcionamiento adecuado del mercado, ya que, esta regulación se justifica por la existencia de factores que incitan prácticas mercantiles ineficientes o inequitativas (fallas de mercado): competencia imperfecta, bienes públicos, monopolios, información incompleta, información asimétrica y externalidades, por lo que allí la regulación se encarga de garantizar las condiciones en las cuales las firmas actúen dentro de un marco de interés común para el funcionamiento del mercado.

La regulación se hace importante, en cuanto interviene para mejorar las fallas de mercado, algunas de estas situaciones son:

- El Monopolio, que consiste en el control de determinado bien o conjunto de bienes por parte $\mathrm{de}$ un solo oferente, éste puede ser natural o creado. Cuando un conjunto de firmas hacen arreglos entre ellas su comportamiento se asimila al monopolio y se denomina cartel.

- Información incompleta o asimétrica, entendida como incompleta cuando no se cuenta con la información necesaria para realizar una transacción. Es asimétrica cuando existe una distribución desigual de la información entre los agentes económicos, oferentes y demandantes, se tiene distinta información acerca de los bienes y servicios existentes en el mercado. Ambas ocasionan una distorsión en el funcionamiento de los mercados no permitiendo una adecuada información.

- Externalidades, que son las consecuencias de las decisiones, acciones $\mathrm{u}$ omisiones de un agente sobre otro agente o un grupo de ellos, estas pueden ser positivas o negativas. Los agentes que generan externalidades negativas generalmente no asumen los costos ocasiona- 
dos por sus acciones, los agentes generadores de externalidades positivas no reciben ninguna recompensa por sus actos, por lo que, según la teoría, no hay una adecuada asignación de recursos en ninguno de los dos casos, haciendo necesario una regulación que no permita este comportamiento o que obligue a los agentes a internalizar el costo de sus acciones.

Por otro lado, el neoinstitucionalismo como lo señala Kalmanovitz $(1997,2003)$ basándose en North \& Thomas (1978) y North (1993) ve el mercado no como algo estático y predeterminado sino como una categoría histórica donde es necesario observar el papel que juegan las instituciones, ya que ellas "sientan las reglas formales e informales de comportamiento de los agentes, establecen derechos de propiedad y sus límites, facilitan y garantizan los contratos privados y públicos, ofrecen información sobre precios y plazos y generan una atmósfera que es conducente o no al desarrollo" (Kalmanovitz, 1997). Donde se introducen problemas no reconocidos por los neoclásicos como "los bienes públicos que son indivisibles y que se financian con impuestos y el problema del poder o sea que los bienes no sólo son producidos sino que también pueden ser tomados o usurpados por otros" (Kalmanovitz, 2003).

Las instituciones son sistemas de reglas que definen el control sobre los medios de producción, el acceso a los recursos, a la información y su control estratégico (De la Garza, 2005). Williamson (2000, 93) considera que "el neoinstitucionalismo trabaja a dos niveles: uno macro, constituido por el medio institucional o las reglas de juego que constriñen las acciones de los agentes y las encausa, y un nivel micro, en el que se dan las instituciones de gobierno sobre los agentes económicos, como son los mercados, los cuasi-mercados y las estructuras jerárquicas de contratación (empresas y burocracias públicas)”.

De esta manera, para la vertiente neoinstitucional el problema es más complejo ya que las instituciones, entendidas como el sistema de reglas que limitan o impulsan la acción del individuo, siendo un resultado histórico que pueden cambiar pero lo hacen lentamente (Kalmanovitz, 1997). Los cambios regulativos sólo se dan en el mediano plazo y debe estar acompañado de cambios sociales que los fortalezcan. $\mathrm{Si}$ bien existen otras visiones que abordan el tema de la regulación ${ }^{1}$, no serán abordadas en este escrito dado su carácter introductorio.

\section{Regulación económica: interés público ó interés privado}

Bajo las perspectivas neoclásica y neoinstitucionalista, la regulación económica es una forma "de intervención pública que impone restricciones, influye o condiciona las conductas o decisiones de los agentes económicos y obliga a que las firmas reguladas se comporten de manera diferente a como actuarían si la regulación no existiera" (Lasheras, 1999 citado en León, 2006, 26). Para abordar el estudio de la regulación económica y el diseño de sus mecanismos, en economía existen dos teorías principales: una enfocada desde el interés público y otra desde el enfoque de los grupos de interés. (León, 2006; Ramírez, 2007).

La teoría del interés público (teoría normativa) se basa en el supuesto de que en sus acciones sobre el sector privado las entidades públicas tratan de maximizar el bienestar social. Imperativo moral basado en la suposición de que existen ciertos intereses públicos (colectivos, comunales o nacionales), a los que se debe servir por encima de los intereses privados (León, 2006; Ramírez, 2007).

León (2006) nos recuerda siguiendo a Mitnick (1974), que el interés público puede referirse a un concepto de carácter "equilibrador donde el interés público resulta a partir de la satisfacción simultánea de aspectos seleccionados de diferentes intereses particulares; negociador, cuando se busca sacrificar algunos intereses particulares para que el resultado

1 Por ejemplo, la escuela de la regulación, de origen francés Centre d'Etudes Prospectives dEconomie Mathématique Appliquées a la Planification (CEPREMAP) y que tiene como principales exponentes a Michel Aglietta, Robert Boyer, Alain Lipietz, entre otros. Esta tradición se abordará en posteriores fases de este trabajo. 
general sea de interés público; intercambio, cuando se pretende que los intereses particulares afectados por la regulación produzcan algún beneficio de interés público, a cambio de ciertos beneficios privados para estos; traslapado de objetivos nacionales o sociales, donde se considera que algunos objetivos sociales o nacionales son de interés público y están por encima de los intereses privados; y paternalista, cuando el interés público se equipara a las preferencias de una persona, grupo u organización." (Mitnick, 1974, citado en León, 2006, 28).

A esta teoría del interés público se le pueden presentar inconvenientes que afecten su objetivo: "que los reguladores se corrompen por la ganancias personales, de tal manera que la regulación que administran se distorsiona para servir a sus intereses personales, la incompetencia de los reguladores y la regulación es capturada por los intereses de reguladores privados." (Lasheras, 1999).

Desde el enfoque del interés privado (teoría positiva) una firma o un conjunto de firmas presionan a los entes reguladores para que legislen a su favor teniendo en cuenta sus propios intereses y factores estratégicos del país o de la región, ejemplo: las privatizaciones, los aranceles, los subsidios, etc.

Aquí existen riesgos como la captura del Estado o del ente regulador por parte de los grupos de interés, lo que afectaría los objetivos que se buscan con dicha regulación. La decisión de intervención de los grupos de interés esta mediada por la racionalidad económica de los agentes, si las retribuciones posteriores a la regulación son superiores a la suma invertida es un fuerte incentivo para hacerlo, sino por lo menos intentarlo.

\section{El problema de la racionalidad}

Comprender la racionalidad de los agentes se hace fundamental para el establecimiento de la regulación en esta perspectiva, ya que lo que se busca es incidir en el comportamiento de estos para lograr un objetivo.

Kalmanovitz (2003) nos recuerda que para los neoclásicos "un individuo se comporta racionalmente frente a una amplia gama de opciones que puede valorar adecuadamente, porque cuenta con una información perfecta" este agente calcula por anticipado los resultados de sus decisiones que son: egoístas e individualistas, donde al buscar el máximo beneficio individual, llevaría hacia el beneficio general. Mientras en el neoinstitucionalismo asume que no es posible que el agente cuente con información perfecta, $y$ tiene limitantes cognitivos que hace que operen de manera equivocada frente a la realidad. A su vez, para los agentes "más importante aún es que las instituciones guían su comportamiento, las normas sociales castigan o aprueban sus acciones, de tal modo que las decisiones económicas óptimas sólo se pueden tomar en ambientes institucionales propicios a la creación de riqueza" (Kalmanovitz, 2003).

La base de esta racionalidad se encuentra en el individualismo metodológico como el que define el camino a seguir en la construcción de teoría, el cual cuenta con unas reflexiones acerca de la realidad y de su comportamiento (De la Garza, 2005; Martínez, 2004; León, 2006):

- Considera que los fenómenos sociales son reducibles a los individuales.

- Utiliza el ceteris paribus es decir la construcción de categorías como si fuera posible el control de variables a semejanza de las Ciencias Naturales en el laboratorio, que conceptualmente fue extendida con los conceptos de "falla de mercado" y de "externalidad".

- Supone al actor como racional (máximizador, calculador y con información suficiente costobeneficio).

- Los individuos toman decisiones racionales con arreglo a fines, teniendo en cuenta sus preferencias (subjetivo) y sus restricciones (objetivo).

- Piensa a la ciencia social a semejanza de las ciencias formales (matemática o lógica), que partiría de axiomas para demostrar teoremas lógicamente impecables a partir de la deducción, que serían verdaderos también empíricamente a condición de que los supuestos se cumplieran en la realidad.

Es decir los principales problemas son la racionalidad de los agentes y la información con que cuentan 
para tomar sus decisiones, sin embargo a este agente racional se le pueden hacer algunas consideraciones como:

- La racionalidad limitada por la que los actores sí buscan optimizar, pero tienen limitaciones cognitivas para lograrlo.

- Las soluciones no son óptimas sino satisfactorias.

- La firma (empresa) no se reduce a una función de producción, sino que es entendida como una estructura organizativa para obtener ganancias.

- Las expectativas no siempre son racionales, sino que son adaptativas.

\section{Relaciones de la regulación contable en términos empresariales}

Las teorías económicas expuestas en este documento parten de un principio inductivo donde se plantea que los fenómenos particulares de la empresa pueden generalizarse en la sociedad, hacen el supuesto que sí es bueno para la "empresa" es bueno para la sociedad. Esto significa que entre más beneficios éstas tengan mayores beneficios tendrá la sociedad. Por ello, la información contable es considerada un bien público, ya que participa en el control de los recursos y la distribución de los beneficios en y desde la empresa, lo que hace importante la regulación en contabilidad para que ésta llegue a su óptimo social tanto en la empresa como en la sociedad (Kaplan, 1991; Beattie, 2004).

Uno de los pilares de la contabilidad especialmente en su corriente dominante es la teoría de la firma (Coase, 1937, 1990), en la que se esbozan los principios de la integración vertical y los costos de transacción, como costos de oportunidad que pueden ser disminuidos por la contabilidad. En el mismo sentido, la teoría de la agencia (Jensen \& Meckling, 1976) busca la disminución de los costos de control entre principales y agentes en las organizaciones -vistas como un conjunto de relaciones contractuales- donde la contabilidad cumple un doble papel: garantizar la ejecución de los contratos y medir las retribuciones de los agentes (Monterrey, 1998; Watts \& Zimmerman, 1986). En este contexto, la contabilidad se entiende "como una función especializada de las firmas, que juega un papel esencial facilitando información en función de la cual se determinan las prestaciones económicas de cada contrato" (Pina, 1988 citado en Monterrey, 1998).

Sunder (2005) presenta argumentos básicos que ayudan a dilucidar la relación entre la contabilidad y el control en las organizaciones. "Para entender la contabilidad y el control en las organizaciones es necesario tener en cuenta tres ideas primordiales. Primera, todas las organizaciones son conjuntos de contratos entre individuos o grupos de individuos. Segunda, el suministro de información común entre las partes contratantes ayuda al diseño y ejecución de estos contratos. Finalmente, el control en la organizaciones es un balance sostenido o un equilibrio entre los intereses de sus participantes" (Sunder, 2005). Entendiendo que, cada individuo o grupo de individuos se denomina agente, el contrato se convierte en un acuerdo formal o informal entre dos o más agentes, estos al perseguir sus propios objetivos dentro de la institución, como en cualquier intercambio económico, generan conflicto o cooperación, que se encuentra determinada por múltiples factores, como son: las reglas y estructura del intercambio; los recursos aportados en balance con los compensaciones recibidas; y por último, la capacidad de los administradores para disminuir los conflictos y aumentar la cooperación.

Así para esta perspectiva, la contabilidad cumple su labor social al coadyuvar en logro de la eficacia de los mercados, que es una condición necesaria para el bienestar social, ya que los demás arreglos se adecuan por medio del sistema de precios bajo el mecanismo de mercado. Donde el establecimiento de normas se da por un proceso político en el que los diferentes grupos de interés (accionistas y directivos) presentan sus intereses en una pugna por el poder para determinarlas (Bettie, 2004). Es decir que lo que es bueno para estos grupos de interés es bueno para la sociedad en su conjunto.

Se hacen importantes las actividades realizadas por la auditoría vista como "una actividad de monitoreo que reduce los problemas de incentivos entre los propietarios y gerentes e incrementa el valor de la firma" (Kaplan, 1991), en el sentido que es de utilidad para los grupos de interés especialmente en la solución de 
las fallas de mercado. La auditoría se realiza como un ejercicio de control a la gestión inicialmente de los propietarios para posteriormente ser de los grupos de interés del ente auditado, enviando señales al mercado -espacio donde se asignan los recursos- $y$ se toman las decisiones por parte de los agentes.

\section{Una visión preponderante de la regulación contable}

La vertiente predominante de la contabilidad, la define como la medición, captación, valoración y representación de hechos económicos en una unidad económica específica (empresa), que debe brindar dentro de la lógica del mercado información necesaria para llevar a cabo las transacciones económicas ya fuesen internas o externas. Así las normas contables "prescriben los criterios específicos de reconocimiento (pautas de registro, clasificación y categorías como noción de activo, p. ej.), medición (criterios de medida y valor, con o sin ajustes por inflación, valores de entrada o valores de salida) y revelación (especificidades de política contable, criterios de amortización, depreciación, y extinción, tasación de diferencias por opciones alternativos, entre otros)". (Gómez, 2007).

Al inicio las prácticas contables fueron moldeadas por aquellos que ejercían la contabilidad y poco a poco por entidades gubernamentales como medida de control. Estas prácticas en un principio respondían a necesidades particulares de las empresas: costos, presupuestos e información para los dueños/ administradores; con el avance de las actividades mercantiles $e$ industriales y el aumento del tamaño de las compañías pasando a convertirse en grandes corporaciones, se crearon nuevas necesidades de información destinada a múltiples usuarios.

Por lo tanto, se puede entender la regulación contable, como la forma de armonizar una práctica social dentro de las condiciones determinadas por el mundo material y social, influenciadas por un conjunto de proposiciones generalmente de origen científico.
En EEUU fue sólo hasta la organización del mercado de valores y la creación de la Securities and Exchange Commision (SEC) que comenzó la promulgación de una normatividad contable para ser utilizada de forma general, Por medio de la promulgación de $\mathrm{Ge}$ nerally Accepted Accounting Principles (GAAP) que deberían ser usados por las compañías principalmente de aquellas que cotizaban en la bolsa de valores.

La mecánica consistió en que la SEC, entidad Estatal, delegó en el gremio de contadores (privado) el American Institute of Certified Public Accountants ${ }^{2}$ (AICPA), quien creó el Committee on Accounting Procedure $^{3}$ (CAP), donde se comenzó por los Accounting Research Bulletins (ARB) basados en la aceptación generalizada (inductivo - positivista), la observación de las mejores prácticas -lo que es-. El CAP fue sustituido por el Accounting Principies Board (APB) que emitía opiniones basadas en una metodología lógico-deductiva (normativista), donde se pretendía un ordenamiento desde arriba -deber ser-. Más adelante el AICPA nombró dos grupos de estudio: uno para el establecimiento de los objetivos de los estados financieros, que dio lugar al informe Trueblood; y el otro para el establecimiento de principios de contabilidad (USGAAP) los Statements of Concepts del Financial Accounting Standard Board (FASB) que fueron el inicio de la idea de un marco conceptual para la contabilidad donde se busca que la norma se acomode a los objetivos de la información contable (teleológico), marco de acción que concuerden con las realidades que se quieren influenciar-deber ser de acuerdo a- (Cañibano \& Gonzalo, 1996; Beattie, 2004).

Según Zeff (1999) el impulso dado a la construcción y consolidación de un marco conceptual en la contabilidad ha estado presente desde los inicios de la regulación contable. Pero es desde 1970 que el espíritu de las normas contables se entiende como un medio para la consecución de objetivos de los diferentes usuarios -información para la toma de decisiones-, el llamado paradigma de la utilidad (Tua Pereda, 1990; Araújo, 1994).

\footnotetext{
2 Este instituto que nació en 1887 con la formación de la American Association of Public Accountants (AAPA), en 1916 cambio su nombre a el American Institute of Accountans ( AIA) y ya en 1957 paso a convertirse en AICPA.

3 Comité de procedimiento contable.
} 
La regulación contable prioriza las necesidades de lo que a su interior se considere como usuario de la información, por ejemplo para EEUU los usuarios principales de la información contable eran los inversores y acreedores ${ }^{4}$ mientras que para el Reino Unido aparte de los inversores y acreedores también se incluida a empleados, el gobierno, clientes, proveedores y al público en general ${ }^{5}$. Así, las concepciones de regulación también cambian, por ejemplo, la regulación en EEUU es de tipo económico, mientras que para el Reino Unido es un proceso social (Bettie, 2004).

En el ámbito internacional fue la creación del Comité de Normas Internacionales de Contabilidad (IASC), la que estableció los parámetros regulativos en la presentación de estados financieros para el mercado público de valores internacional. Tiene su origen en 1973 a través de un acuerdo realizado por representantes de profesionales de contabilidad de varios países: Australia, Canadá, Francia, Alemania, Japón, México, los Países Bajos, el Reino Unido e Irlanda, y los Estados Unidos de América. Desde 1982 los "miembros" patrocinadores del IASC comprenden todos los representantes profesionales de la contabilidad que eran miembros de la Federación Internacional de Contadores -International Federation of Accountants IFAC-.

Es decir, que los principios de regulación que han sido liderados en EEUU por el (FASB) de corte económico y en pro del mercado (especialmente el de capitales) se enmarca en una visión de la economía neoclásica mientras que los estándares emitidos por el Internacional Accounting Standard Board (IASB) obedecen a una corriente de tipo neoinstitucionalista de la economía.

En el presente siglo, más precisamente en septiembre de 2002 se firmó el acuerdo Norwalk en virtud del cual el IASB y el FASB, emisores de normas contables, se comprometen a trabajar conjuntamente para acelerar el proceso de convergencia de sus normas, de manera que se consensue en un período de tiempo relativamente corto, un único juego de normas contables para uso global. Proceso que no se ha estado exento de problemas, por ejemplo, todavía en 2011 lo referido "a la normativa aplicable a los instrumentos financieros: mientras el FASB se inclina por el uso generalizado del valor razonable, con algunas excepciones, el IASB opta por mantener un modelo mixto de coste amortizado y valor razonable" (Cañibano \& Herranz, 2011). Es importante recordar que la Unión Europea "únicamente obliga a utilizar las normas del IASB para los estados financieros consolidados de las empresas que cotizan en bolsa, dejando a la decisión de los Estados miembros la posibilidad de extender o no el uso de dichas normas para los restantes estados financieros emitidos por sus empresas" (Cañibano \& Herranz, 2011).

En la UE debido a los objetivos y necesidades particulares de cada país miembro, elijen la normatividad a aplicarse para la presentación de los siguientes estados financieros: i) estados financieros individuales de las empresas que cotizan en bolsa; ii) estados financieros consolidados de las empresas que no cotizan en bolsa; y iii) estados financieros individuales de las empresas que no cotizan en bolsa. Como lo señala Cañibano \& Herranz (2011) la discrecionalidad en la solución de que normatividad aplicar ha generado una dispersión de criterios.

Aquí es importante resaltar que en la UE existe una gran diversidad de grupos de interés que entran en el juego político de la implantación de normas, lo que permite que la contabilidad intente responder a todos ellos en sus procesos contables y crea otro tipo de informes, además del financiero, para una mejor toma de decisiones, por ejemplo el Global Reporting Initiative (GRI).

Con los problemas sociales, ambientales y económicos se hace insuficiente para la contabilidad enfocarse únicamente en la transmisión de información como bien público ya que los intereses que existen sobre su actuación exceden a la simple medición de flujos de caja, se comienza a hacer necesario considerar si la contabilidad erige reglas (formales $e$ informales) que incidan en el comportamiento de los

\footnotetext{
Informe Trueblood
}

The Corporate Report. 
agentes, establece derechos de propiedad, facilita y garantiza los contratos privados y públicos, ofrece dispositivos de información sobre precios y plazos, y coadyuva a generar una atmósfera que es conducente desarrollo. En otras palabras, ¿es la contabilidad una institución?, parece ser ésta la pregunta que se debe responder para continuar con el tema de la regulación contable desde la perspectiva de la economía ortodoxa.

Unido a lo anterior, se debe ponderar de acuerdo al contexto de aplicación: las necesidades de información, los grupos de interés, la formación de los agentes que toma de decisiones y evidenciar la presión de los agentes privados en la configuración de las normas contables. Dicho análisis debe partir necesariamente de visiones alternativas que permitan introducir cambios paulatinos en la práctica contable.

\section{Conclusiones}

La regulación contable tiene un trasfondo teórico que se enmarca en los desarrollos del modelo neoclásico y neoinstucionalista, dilucidar sus fundamentos permite comprender los objetivos de la regulación y vincularlos con las metas económicas y sociales que tengan los países. Vincular la contabilidad como una institución permitiría enmarcarla mucho mejor dentro de la teoría y ayudaría para el análisis de las normas contables y sus efectos.

Se hace necesario reconocer que los usuarios de la información contable son mucho más que los accionistas, inversionistas y acreedores, extendiéndose a otros diversos grupos de interés permitiendo evidenciar las necesidades reales del contexto donde se va a aplicar la regulación contable.

Al presentarse objetivos económicos y sociales distintos, acordes a las necesidades de cada país, los marcos regulativos contables deben corresponder entonces con la consecución de estos. En este punto, el marco teórico que nos presenta la teoría neoclásica y neoinstitucional es insuficiente para dilucidar las implicaciones económicas y sociales de la regulación contable.

\section{Referencias}

Araújo, E. (1994). Un nuevo paradigma contable para Colombia: la utilidad de la información -o el Decreto 2649 de 1993-. En: Revista Contaduría Universidad de Antioquia, 24-25: 127-137.

Beattie, V. (2004). Tradiciones de investigación en contabilidad financiera. En: Ryan, B. (eds.). Metodología de la investigación en Finanzas y contabilidad (p.p. 129-154). Barcelona: Ediciones Deusto.

Cañibano, L. \& Herranz, F. (2011). La "otra contabilidad" y sus efectos económicos. En: Revista de buen gobierno y responsabilidad Corporativa, 57.

Cañibano, L. \& Gonzalo, J. (1996). Los programas de investigación en contabilidad. En: Revista Contaduría Universidad de Antioquia, 29: 12-53.

Cataño, J. (2001). ¿Por qué el predominio de la teoría neoclásica? En: Cuadernos de Economía, XX (34): 281-291.

Coase, R. (1937). The nature of the firm. En: Economica, 9: 386-405.

Coase, R. (1990). Accounting and the Theory of the Firm. En: Journal of Accounting and. Economics, 12: 3-13.

De la Garza, E. (2005). El Neoinstitucionalismociopción ante la elección racional? Una discusión entre la Economía y la Sociología. En: Revista Mexicana de Sociología, 67(1): 163-203.

Gómez, M. (2007). Comprendiendo las relaciones entre los sistemas contables, los modelos contables y los sitemas de información contables empresariales. En: Revista internacional Legis de contabilidad \& auditoría, 32: 83-113.

Greenwald, B. \& Stiglitz, J. (1986). Externalities in economies with imperfect information and incomplete markets. En: Quarterly Journal of Economics, 90: 229-264.

Jensen, M. \& Meckling, W. (1976). Theory of the Firm: Managerial Behavior, Agency Costs and Ownership Structure. En: Journal of Financial Economics, 3: 305-360.

Kalmanovitz, S. (1997). Las instituciones, la ley y el desarrollo económico. En borradores Semanales de Economía (B. de la R.), 69.

Kalmanovitz, S. (2003). El Neoinstitucionalismo como escuela. En: Revista de Economía Institucional, 5 (9): 189-212.

Kaplan, S. (1991). Positive theory, rationality and accounting regulation. En: Critical Perspectives on Accounting, 2 (4): 361-374.

Krugman, P. (2009). El Retorno de la Economía de la Depresión. Barcelona: Editorial Crítica.

Lasheras, M. (1999). La regulación económica de los servicios públicos. Editorial Ariel S.A, Barcelona.

León, N. (2006). Mecanismos para la regulacion ambiental. Universidad Nacional de Colombia, Bogotá.

Martínez, J. (2004). Tipos de elección racional. En: Revista Internacional de Sociología, 37: 139-173.

Mitnick, B. (1974). "The Sitting Impasse and Rationa Choice Model of Regulatory Behavior: An agency for Power Plant Sitting". En: Journal of Environmental and Management, 1: 150-171.

Monterrey, J. A. (1998). Un recorrido por la contabilidad positiva. En: Revista Española de Financiación y Contabilidad, XXVII (95): 427-467.

North, D. (1993). Instituciones, cambio institucional y desempeño económico. Fondo de Cultura Económica, México.

North, D. \& Thomas, R. (1978). El nacimiento del mundo occidental: una nueva historia económica (900-1700). Siglo Veintiuno Editores, México. 
Ramírez, H. (2007). La política de competencia y el proceso de regulación en México. Edición electrónica. Texto completo en: www.eumed.net/libros/2007b/281/

Stiglitz, J. (2006). Cómo hacer que funcione la globalización. Altea Taurus Alfaguara, México.

Sunder, S. (2005). Teoría de la contabilidad y el control. Universidad Nacional de Colombia, Bogotá.

Tua Pereda, J. (1990). Algunas Implicaciones del Paradigma de Utilidad en la Disciplina Contable. En: Revista Contaduría Universidad de Antioquia, 16: 17-39.
Watts, R. \& Zimmerman, J. (1986). Positive Accounting Theory. Prentice Hall, México..

Williamson, O. (2000). "Economic institutions and development: a view from the bottom". En: Olson, M. \& Kähköhnen, S. (eds). A Not-so-dismal Science: A Broader View of Economies and Societies. Oxford Scholarship Online.

Zeff, S. (1999). La evolución del marco conceptual para las empresas mercatinles en los Estados Unidos. En: Revista Española de financiación y contabilidad, 100: 151-194. 